\title{
Long-term evolution of FU Orionis objects at infrared wavelengths ${ }^{\star}$
}

\author{
P. Ábrahám ${ }^{1}$, Á. Kóspál ${ }^{2}$, Sz. Csizmadia ${ }^{1}$, M. Kun ${ }^{1}$, A. Moór ${ }^{1}$, and T. Prusti ${ }^{3}$ \\ 1 Konkoly Observatory of the Hungarian Academy of Sciences, PO Box 67, 1525 Budapest, Hungary \\ e-mail: abraham@konkoly.hu \\ 2 Department of Astronomy, Eötvös Loránd University, PO Box 32, 1518 Budapest, Hungary \\ ${ }^{3}$ ESTEC/SCI-SAF, Postbus 299, 2200 AG Noordwijk, The Netherlands
}

Received 23 February 2004 / Accepted 26 July 2004

\begin{abstract}
We investigate the brightness evolution of seven FU Orionis systems in the 1-100 $\mu$ m wavelength range using data from the Infrared Space Observatory (ISO). The ISO measurements were supplemented by 2MASS and MSX observations performed in the same years as the ISO mission (1995-98). The spectral energy distributions (SEDs) based on these data points were compared with earlier ones derived from the IRAS photometry as well as from ground-based observations carried out around the epoch 1983. In three cases (Z CMa, Parsamian 21, V1331 Cyg) no difference between the two epochs was seen within the measurement uncertainties. V1057 Cyg, V1515 Cyg and V1735 Cyg have become fainter at near-infrared wavelengths while V346 Nor has become slightly brighter. V1057 Cyg exhibits a similar flux change in the mid-infrared. At $\lambda \geq 60 \mu \mathrm{m}$ most of the sources remained constant; only V346 Nor seems to fade. Our data on the long-term evolution of V1057 Cyg agree with the model predictions of Kenyon \& Hartmann (1991) and Turner et al. (1997) at near- and mid-infrared wavelengths, but disagree at $\lambda>25 \mu \mathrm{m}$. We discuss if this observational result at far-infrared wavelengths could be understood in the framework of the existing models.
\end{abstract}

Key words. stars: pre-main sequence - stars: circumstellar matter - stars: individual: V1057 Cyg - infrared: stars

\section{Introduction}

FU Orionis objects are low mass pre-main sequence stars defined as a class by Herbig (1977). Most members of this class have undergone outbursts in optical light of 4 mag or more, followed by a fading phase on the timescale of several decades. In some objects (e.g. ZCMa, L1551 IRS 5, BBW 76) eruption in the optical was not observed but they were identified as class members on the basis of their spectral characteristics. For a comprehensive review of FU Orionis objects see Hartmann \& Kenyon (1996).

According to the most widely accepted picture the FU Orionis outburst is a consequence of a rapid temporal increase of the disk accretion rate (Hartmann \& Kenyon 1996). Predictions of these types of models (e.g. Kenyon \& Hartmann 1991; Bell et al. 1995; Turner et al. 1997) have to be confronted with multiwavelength monitoring observations of the outburst period and the fading phase. The fading phase is well documented in the optical/near-infrared (Kopatskaya 1984; Ibragimov \& Shevchenko 1988; Kenyon \& Hartmann 1991). Very few data have been available so far at mid- and far-infrared wavelengths where thermal emission of the disk

^ Based on observations with ISO, an ESA project with instruments funded by ESA member states (especially the PI countries France, Germany, the Netherlands and the United Kingdom) with participation of ISAS and NASA. and the circumstellar envelope can be observed. Recently the Infrared Space Observatory (ISO, 1995-98, Kessler et al. 1996) provided new photometric data in the $4.8-200 \mu \mathrm{m}$ range on FU Orionis-type stars.

In our study we search for systematic brightness variations during the post-outburst phases of 7 FU Orionis objects at mid- and far-IR wavelengths. We compiled two SEDs for each object: the first is based on IRAS photometry as well as groundbased observations and is representative of the evolutionary status around 1983. The second SED was compiled from observations taken around 1996-2000 including data from ISOPHOT (the infrared photometer on-board ISO, Lemke et al. 1996), MSX (Egan \& Price 1996), and 2MASS (Cutri et al. 2003). The comparison of the two SEDs provides information on the wavelength dependence of the far-infrared flux evolution during a period of 15 years. As an example in the case of V1057 Cyg we present a detailed comparison of our results with the predictions of the models of Kenyon \& Hartmann (1991) and Turner et al. (1997).

\section{Target list, observations and data reduction}

\subsection{Up-to-date catalogue of FU Orionis objects}

When Herbig (1977) defined the class of FU Orionis objects, there were only three known members. Since then a number 
Table 1. Up-to-date catalogue of FU Orionis objects (for column description see Sect. 2.1). References: [1] Eislöffel et al. (1990); [2] candidate, other designation: V1184 Tau, Yun et al. (1997); [3] Herbig (1977); [4] Carr et al. (1987); [5] Sandell \& Aspin (1998); [6] Staude \& Neckel (1992); [7] Reipurth 50 N IRS 1, Strom \& Strom (1993), $\alpha_{2000}=5^{\mathrm{h}} 40^{\mathrm{m}} 27.4^{\mathrm{s}} \delta_{2000}=-7^{\circ} 27^{\prime} 33^{\prime \prime}$; [8] assumed to be in a pre-outburst state, McMuldroch et al. (1992); [9] Elias (1978); [10] Graham \& Frogel (1985); [11] Hartmann et al. (1989); [12] Staude \& Neckel (1991); [13] Kenyon et al. (1993); [14] Hodapp (1995); [15] Hartmann \& Kenyon (1996); [16] Aspin \& Sandell (2001); [17] Reipurth \& Aspin (1997); [18] Sandell \& Weintraub (2001); [19] Chavarria-K. (1981); [20] Eiroa \& Hodapp (1990); [21] Herbst et al. (1978); [22] candidate, Aspin \& Reipurth (2003); [23] de Lara et al. (1991); [24] FU Ori candidate, in the state of rapid brightening since January 2004. An associated submm source was detected by Mitchell et al. (2001); [25] Lis et al. (1999).

\begin{tabular}{|c|c|c|c|c|c|c|}
\hline Object & Outburst & $D[\mathrm{pc}]$ & IRAS & MSX & ISO & 2MASS \\
\hline $\mathrm{BBW} 76^{1}$ & $<1930^{15}$ & $1700^{18}$ & $07486-3258$ & G248.7075-03.3686 & & $07503560-3306238$ \\
\hline CB34 $\mathrm{V}^{2}$ & $<1994^{2}$ & $1500^{18}$ & & G186.9520-03.8325 & & $05470377+2100347$ \\
\hline $\mathrm{FU} \mathrm{Ori}^{3}$ & $1937^{15}$ & $450^{18}$ & $05426+0903$ & & SWS, LWS & $05452235+0904123$ \\
\hline V1057 $\mathrm{Cyg}^{3}$ & $1970^{15}$ & $600^{18}, 700^{19}$ & & G085.4595-01.0468 & PHT, LWS & $20585371+4415283$ \\
\hline V1515 Cyg $^{3}$ & $1950 \mathrm{~s}^{15}$ & $1000^{18}, 1050^{3}$ & $20220+4202$ & G079.9187+02.7391 & PHT & $20234802+4212257$ \\
\hline L1551 IRS $5^{4}$ & $?$ & $140^{18}$ & $04287+1801$ & & & $04313407+1808049$ \\
\hline $\mathrm{PP} 13 \mathrm{~S}^{5}$ & $<1900^{16}$ & $300^{18}$ & $04073+3800$ & & & $04104088+3807517$ \\
\hline Parsamian $21^{6}$ & $?$ & $1800^{18}, 400^{20}$ & $19266+0932$ & G045.8149-03.8309 & PHT & $19290085+0938429$ \\
\hline $\operatorname{Re} 50 \mathrm{NIRS} 1^{7}$ & $1960-70^{17}$ & $460^{18}$ & & & PHT, SWS & $?$ \\
\hline V1331 $\mathrm{Cyg}^{8}$ & $?$ & $550^{18}, 700^{19}$ & $20595+5009$ & G090.3121+02.6774 & LWS & $21010920+5021445$ \\
\hline V1735 Cyg $^{9}$ & $1957-65^{15}$ & $900^{18}$ & $21454+4718$ & G093.7587-04.6371 & PHT, LWS, SWS & $21472065+4732035$ \\
\hline V346 Nor ${ }^{10}$ & $\sim 1984^{15}$ & $700^{18}$ & $16289-4449$ & G338.5458+02.1178 & CAM, PHT, LWS, SWS & $16323219-4455306$ \\
\hline V883 Ori $^{7}$ & $?$ & $460^{18}$ & 05358-0704 & & & $05381810-0702259$ \\
\hline $\mathrm{ZCMa}^{11}$ & $?$ & $930^{18}, 1150^{21}$ & 07013-1128 & G224.6077-02.5574 & PHT, LWS, SWS & 07034316-1133062 \\
\hline RNO $1 B^{12}$ & $?$ & $850^{18}$ & $00338+6312$ & G121.2940+00.6572 & CAM, LWS, SWS & $00364599+6328529$ \\
\hline RNO $1 C^{13}$ & $?$ & $850^{18}$ & $00338+6312$ & G121.2940+00.6572 & CAM, LWS, SWS & $00364659+6328574$ \\
\hline $\mathrm{AR} 6 \mathrm{~A}^{22}$ & $?$ & $800^{22}$ & & $\mathrm{G} 203.2028+02.0653$ & & $06405930+0935523$ \\
\hline $\mathrm{AR} 6 \mathrm{~B}^{22}$ & $?$ & $800^{22}$ & & G203.2028+02.0653 & & $06405930+0935523$ \\
\hline OO Ser ${ }^{14}$ & $1995^{14}$ & $311^{23}$ & & & CAM, PHT, LWS, SWS & $18294913+0116206$ \\
\hline V1647 Orii ${ }^{24}$ & 2004 & $400^{25}$ & 05436-0007 & & CAM & 05461313-0006048 \\
\hline
\end{tabular}

of new objects have been identified. As a first step of our study we compiled an up-to-date list of FU Ori stars. We included FU Ori candidates without attempting to homogenise the identification criteria of different authors. The resulting catalogue, which is to a large extent similar to the list of Sandell \& Weintraub (2001), is presented in Table 1. In this table Col. 1 refers to the original paper where the object was identified as FU Ori type. The next two columns give the date of outburst (if known) and the distance to the object, respectively. Associated sources in the IRAS and MSX catalogues are given only in the case of good positional coincidence with the optical coordinates. The RNO 1B/1C system (separation $\sim 5^{\prime \prime}$ ) was detected but unresolved by both IRAS and MSX. Column 6 shows which of the four ISO instruments (ISOCAM, ISOPHOT, ISO-LWS, ISO-SWS) observed the source. These observations are available for the public in the ISO Data Archive ${ }^{1}$. The last column identifies the corresponding sources in the nearinfrared 2MASS survey. These source names were derived from the sexagesimal coordinates, and give the equatorial positions for the epoch 2000. (For the position of Re 50 N IRS1 see figure caption.)

\footnotetext{
1 www.iso.vilspa.esa.es/ida/
}

\subsection{Observations}

Table 2 lists the sources of infrared photometric data used in our study. The time distribution of the active periods of the instruments/projects provides a possibility to check for longterm variations of the infrared fluxes between $~ 1983$ (IRAS, ground-based data) and 1996-2001 (ISO, MSX, 2MASS).

From the sources in Table 1 we selected those 7 objects for further study where enough data were available at both epochs ( 1983 and 1996-2000). Five of them have ISO multifilter observations to create a complete mid/far-infrared SED. One object, Parsamian 21, was measured with ISOPHOT only at 65 and $100 \mu \mathrm{m}$, but MSX data are available at shorter wavelengths. V1331 Cyg was not observed by ISOPHOT, but the availability of MSX fluxes makes possible the study of temporal variation at $\lambda \leq 25 \mu \mathrm{m}$. The detailed log of ISOPHOT observations is presented in Table 3.

\subsection{ISOPHOT data processing}

Most observations were carried out as pairs of $\mathrm{ON}$ and OFF measurements separated by $6-12^{\prime}$. The $180^{\prime \prime}$ aperture was selected because it was comparable to the IRAS far-infrared 
Table 2. Sources of infrared photometric data used in our study.

\begin{tabular}{lllc}
\hline \hline Instrument & Wavelengths $[\mu \mathrm{m}]$ & Aperture & Active period \\
\hline Ground-based & $J H K L M N Q$ & $\leq 6^{\prime \prime}$ & $1970 \mathrm{~s}-$ \\
IRAS & $12,25,60,100$ & $1-3^{\prime}$ & 1983 \\
MSX & $4.25,4.29,8.28$ & $18^{\prime \prime}$ & $1996-1997$ \\
& $12.13,14.65,21.34$ & & \\
2MASS & $J, H, K \mathrm{~s}$ & & $1997-2001$ \\
ISOPHOT & $4.8-120$ & $43^{\prime \prime}-180^{\prime \prime}$ & $1995-1998$ \\
\hline
\end{tabular}

beam size. The far-infrared flux of Z CMa was extracted from a $5 \times 1$ scan obtained with the $\mathrm{C} 100$ camera. Three objects were measured at 65 and $100 \mu \mathrm{m}$ in the high-resolution PHT32 mapping mode (see The ISO Handbook vol. V, Laureijs et al. 2001).

The data reduction was performed using the ISOPHOT Interactive Analysis Software Package V10.0 (PIA, Gabriel et al. 1997). After corrections for non-linearities of the integration ramps, the signals were transformed to a standard reset interval. Then an orbit-dependent dark current was subtracted and cosmic ray hits were removed. If the signal did not fully stabilise during the measurement time due to detector transients, only the last part of the data stream was used. This was found mainly in observations with the $4.8 \mu \mathrm{m}$ and $12 \mu \mathrm{m}$ filters, while at other wavelengths the measurements showed sufficient stability. The calibration of measurements at $4.8,12$, and $25 \mu \mathrm{m}$ was performed by adopting the default responsivity. At longer wavelengths we first tried comparison with the on-board fine calibration source (FCS); however, in most ON/OFF observations the quality of the FCS measurement was unacceptable and we returned to the default responsivity. As error estimate we adopted an absolute calibration uncertainty of $25 \%$, which represents well the sum of the random and systematic uncertainties. Colour corrections were applied for each measurement by convolving the observed SED with the ISOPHOT filter profile in an iterative way.

Three objects (V1057 Cyg, V1735 Cyg, Parsamian 21) were measured at 65 and $100 \mu \mathrm{m}$ in the PHT32 observing mode (oversampled map). Since this method is not scientifically validated we used our own procedure for evaluation. Instead of the highly interactive tool implemented in PIA (Tuffs \& Gabriel 2003) we evaluated 23 observations of ISOPHOT standards (stars, asteroids, and planets) with default processing and derived an empirical correction curve from the comparison of the measured fluxes with their model predictions. The fluxes were extracted from the maps using an algorithm like the one developed for the ISOPHOT mini-map observing mode (Moór et al. 2004). The typical uncertainty of the empirical correction does not exceed the adopted $25 \%$ error.

\subsection{IRAS data}

In order to have solid IRAS photometry for comparison with the ISOPHOT data we have re-analysed the IRAS raw data of two FU Orionis objects where the IRAS fluxes published by different authors (PSC, Weaver \& Jones 1992; Kenyon \& Hartmann 1991) were discrepant. We utilised the SCANPI processing tool at IPAC ${ }^{2}$. The results are listed in Table 4; remarks on individual objects are given below.

V1057 Cyg. This source was at the very edge of the IRAS coverage and only a few scans are available. At $12-60 \mu \mathrm{m}$ we adopted the fluxes published by Weaver \& Jones (1992), though their formal uncertainties seem to be too optimistic. At $100 \mu \mathrm{m}$, where only two detectors crossed the star, the source is surrounded by structures which are equally strong or stronger than V1057 Cyg itself. The flux uncertainty is fully dominated by the accuracy of the background determination. Experimenting with different background subtractions we estimate $47 \pm 10$ Jy for V1057 Cyg's flux density at $100 \mu \mathrm{m}$.

V1515 Cyg. In this case the background is even more structured than around V1057 Cyg, and the coarse spatial resolution of IRAS is a problem already at $12 \mu \mathrm{m}$. At 12 and $25 \mu \mathrm{m}$ we estimate $3.7 \pm 1.0 \mathrm{Jy}$ and $6.8 \pm 2.1 \mathrm{Jy}$, respectively, as integrated flux densities which also include an extended structure around the source, probably the infrared equivalent of the ring one can see in the optical wavelengths. The formal errors reflect the uncertainty related to the background subtraction. At $60 \mu \mathrm{m}$ the lack of spatial resolution is the limiting factor. The emission peak is offset by about $20^{\prime \prime}$ from the nominal position of V1515 Cyg, indicating that part of the emission from the region is unrelated to the star. For the flux density towards the position of V1515 Cyg our estimate is $25 \pm 10 \mathrm{Jy}$. At $100 \mu \mathrm{m}$ the emission is dominated by a local peak at about $1^{\prime}$ from the position of V1515 Cyg, and only an upper limit of $110 \mathrm{Jy}$ can be derived for the FU Orionis object.

\section{Results}

The results of the ISOPHOT photometry as well as our newly derived IRAS flux densities are presented in Table 4. Figure 1 displays the SEDs of the sources from the two different epochs ( 1983 and 1996-2000). The results for V1057 Cyg, supplemented with optical data, are shown in Fig. 2.

By comparing the two SEDs of each source one can see the following evolutionary trends:

- at near-IR wavelengths $(\lambda \leq 5 \mu \mathrm{m})$ the sources show a variety of behaviour. Parsamian 21, V1331 Cyg and ZCMa are unchanged, V1057 Cyg, V1515 Cyg and V1735 Cyg have faded, V346 Nor has become slightly brighter;

- in the mid-IR $(5 \leq \lambda \leq 20 \mu \mathrm{m})$ only V1057 Cyg shows a systematic flux change: it has become fainter by a factor of 2 during the period (lower panel in Fig. 2);

- at far-IR wavelengths $(\lambda \geq 60 \mu \mathrm{m})$ five stars (V1057 Cyg, V1735 Cyg, ZCMa, Par 21, and V1515Cyg) remained constant while V346 Nor seems to have become fainter. For V1331 Cyg there are no FIR data other than IRAS.

Below we make remarks on the individual sources. The case of V1057 Cyg will be discussed in detail in Sect. 4.

\footnotetext{
${ }^{2}$ http://irsa.ipac.caltech.edu/applications/Scanpi/
} 
Table 3. Log of ISOPHOT observations. Observing modes are described in Sect. 2.3, ISO_id is the unique 8-digit identification number of ISO observations.

\begin{tabular}{lccccl}
\hline \hline Object & Wavelengths $[\mu \mathrm{m}]$ & Aperture ["] & Date & Obs. mode & ISO_id \\
\hline V1057 Cyg & $4.8,12,25,60,100$ & 180 & 17-Apr.-1996 & ON/OFF & $15200607 / 15200608$ \\
& 65,100 & $43 \times 43$ & 8-Nov.-1996 & PHT32 & 35800611 \\
\hline V1515 Cyg & $4.8,12,25,60$ & 180 & 24-Apr.-1996 & ON/OFF & $15900605 / 15900606$ \\
& 120 & $180 \times 180$ & 24-Apr.-1996 & ON/OFF & $15900613 / 15900614$ \\
\hline Parsamian 21 & 65,100 & $43 \times 43$ & 28-Sep.-1996 & PHT32 & 31601103 \\
\hline V1735 Cyg & $4.8,12,25,60,100$ & 180 & 21-Apr.-1996 & ON/OFF & $15600909 / 15600910$ \\
& $4.8,12,25,60,100$ & 180 & 20-May-1996 & ON/OFF & $18501409 / 18501410$ \\
& 65,100 & $43 \times 43$ & 3-Dec.-1996 & PHT32 & 38301012 \\
\hline V346 Nor & $4.8,12,25,60,100$ & 180 & 9-Feb.-1996 & ON/OFF & $08402303 / 08402304$ \\
\hline ZCMa & $4.8,12,25$ & 52 & 5-Nov.-1997 & ON/OFF & $72003006 / 72003005$ \\
& 60,100 & 99 & 5-Nov.-1997 & $5 \times 1$ scan & 72003007 \\
\hline
\end{tabular}
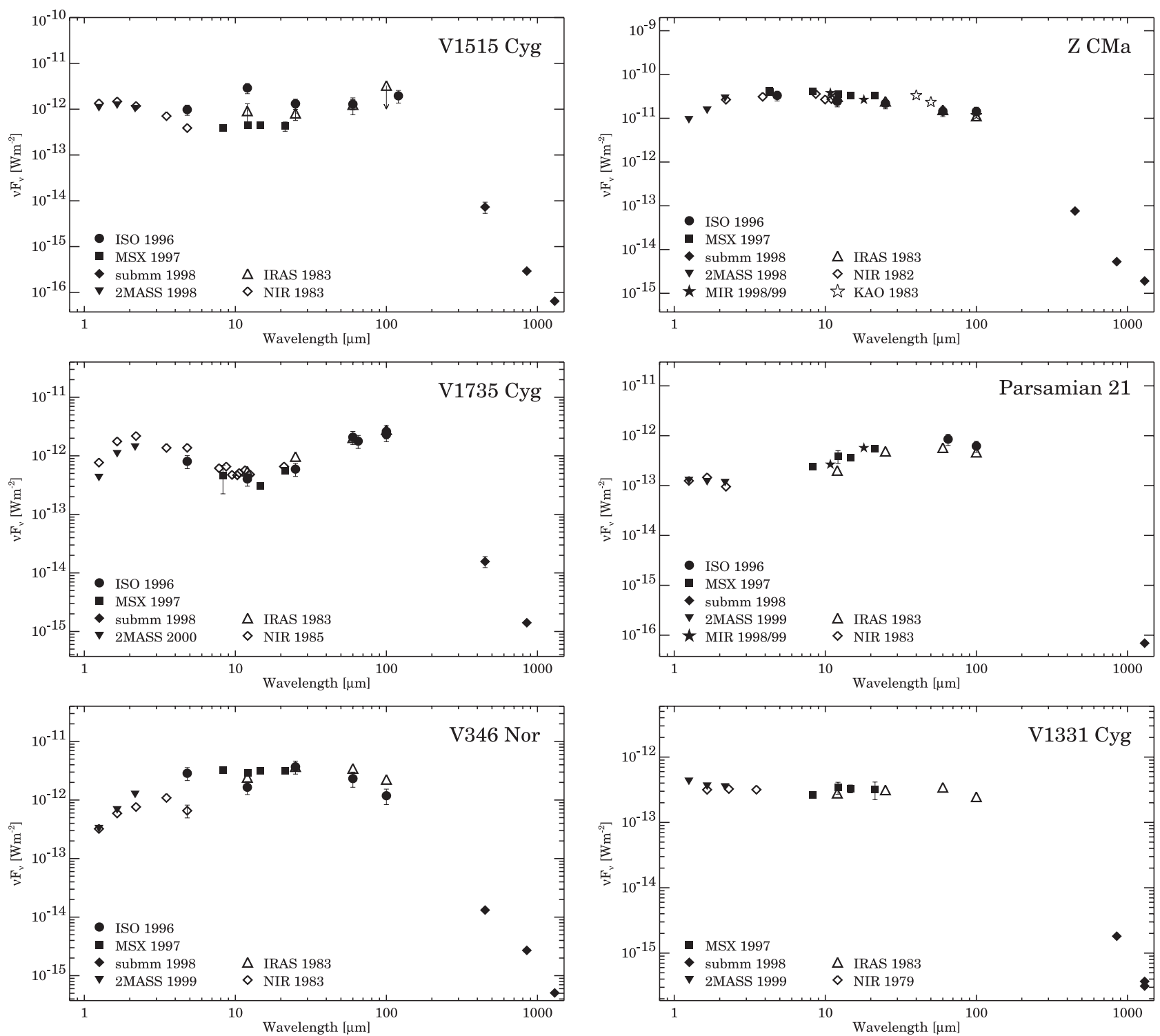

Fig. 1. Spectral energy distributions of 6 FU Orionis-type objects. Open symbols correspond to the epoch of $\sim 1983$ while filled symbols mark the SED as of 1996-2000. The data are presented with no reddening correction. Error bars smaller than the symbol size were not plotted. For V1331 Cyg there are no NIR data from 1983; we plotted data from 1979 (the $K$-band lightcurve shows constant flux in this period). In the case of V346 Nor the ISO and IRAS beams also include the nearby pre-main sequence star Reipurth 13. For comparison the plotted MSX flux densities are sums of the individual fluxes of the two objects. 
Table 4. Photometric results of ISOPHOT and IRAS. All fluxes are colour-corrected and presented in Jy. (*): PHT32 observing mode. Source of IRAS data: PSC - V1735 Cyg, V346 Nor, Z CMa, Parsamian 21; Weaver \& Jones (1992) - V1331 Cyg; this work (Sect. 2.4) - V1515 Cyg. In the case of V1057 Cyg the 12-60 $\mu \mathrm{m}$ fluxes are from Weaver \& Jones (1992), the flux at $100 \mu \mathrm{m}$ was re-determined in this work (Sect. 2.4).

\begin{tabular}{cccccccc}
\hline \hline$\lambda[\mu \mathrm{m}]$ & V1057 Cyg & V1515 Cyg & V1735 Cyg & V346 Nor & ZCMa & Parsamian 21 & V1331 Cyg \\
\hline 4.8 & $2.67 \pm 0.67$ & $1.57 \pm 0.39$ & $1.29 \pm 0.32$ & $4.59 \pm 1.15$ & $53.16 \pm 13.29$ & & \\
12 & $5.68 \pm 1.42$ & $11.64 \pm 2.91$ & $1.62 \pm 0.40$ & $6.61 \pm 1.65$ & $97.33 \pm 24.33$ & & \\
25 & $23.19 \pm 5.80$ & $11.01 \pm 2.75$ & $4.94 \pm 1.24$ & $30.82 \pm 7.71$ & $182.8 \pm 45.7$ & & \\
60 & $52.99 \pm 14.11$ & $25.80 \pm 6.45$ & $41.76 \pm 10.44$ & $46.54 \pm 13.45$ & $290.1 \pm 72.5$ & & \\
$65^{*}$ & $42.26 \pm 10.57$ & & $38.68 \pm 9.67$ & & & $18.51 \pm 4.63$ & \\
100 & $34.50 \pm 9.28$ & & $77.23 \pm 19.31$ & $39.52 \pm 11.65$ & $479.1 \pm 119.8$ & & \\
$100^{*}$ & $62.06 \pm 15.52$ & & $86.82 \pm 21.71$ & & & $20.71 \pm 5.18$ & \\
120 & & $78.45 \pm 24.03$ & & & & & \\
\hline IRAS-12 & $14.89 \pm 0.11$ & $3.7 \pm 1.0$ & $2.19 \pm 0.20$ & $9.73 \pm 0.39$ & $125.1 \pm 5.0$ & $0.80 \pm 0.06$ & $1.12 \pm 0.03$ \\
IRAS-25 & $28.72 \pm 0.08$ & $6.8 \pm 2.1$ & $8.09 \pm 0.52$ & $30.98 \pm 1.33$ & $204.5 \pm 8.7$ & $4.07 \pm 0.26$ & $2.62 \pm 0.02$ \\
IRAS-60 & $53.71 \pm 2.48$ & $25 \pm 10$ & $40.84 \pm 4.90$ & $69.07 \pm 4.83$ & $312.3 \pm 40.6$ & $11.49 \pm 1.03$ & $6.88 \pm 0.23$ \\
IRAS-100 & $47.0 \pm 10.0$ & $<110$ & $92.97 \pm 15.63$ & $74.91 \pm 4.20$ & $375.5 \pm 63.2$ & $15.58 \pm 2.04$ & $8.22 \pm 1.19$ \\
\hline
\end{tabular}

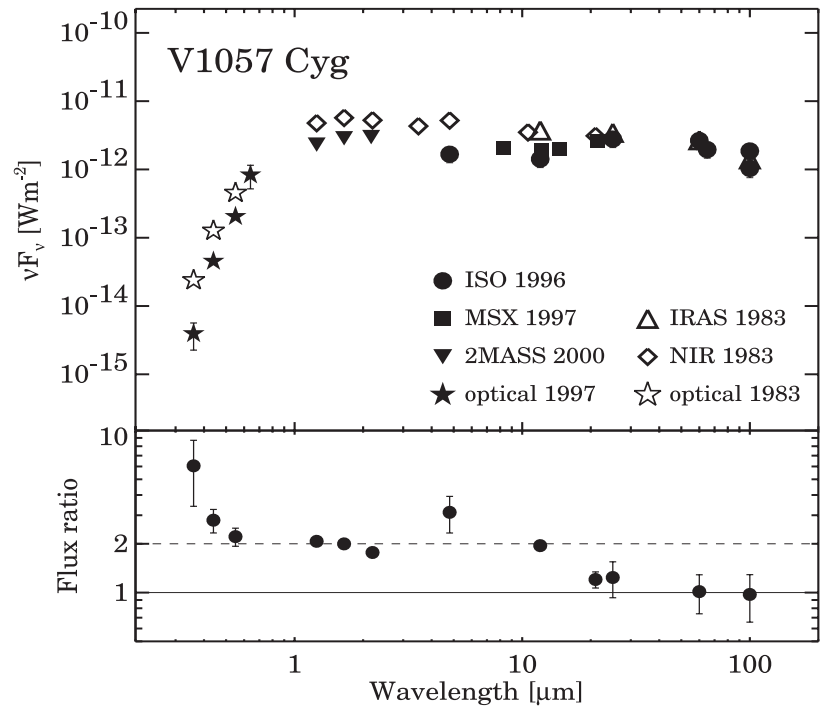

Fig. 2. Spectral energy distribution of V1057 Cyg. Open and filled symbols are as in Fig. 1. No reddening correction was performed. The lower panel shows the ratios of fluxes obtained at the two epochs ( 1983 to 1996-2000).

V1515Cyg. According to the $850 \mu \mathrm{m}$ submillimetre map of Sandell \& Weintraub (2001) this source is situated in the middle of a cold arcminute-size core, and this extended emission is also visible on the MSX $8.28 \mu \mathrm{m}$ map. In addition the 2MASS and MSX data reveal a compact object, 2MASS 20235198+4211260, at about 80" from V1515 Cyg. Since this source is not included in the MSX Point Source Catalog, we extracted fluxes from the MSX maps using aperture photometry. The resulting SED peaks at $\approx 4 \mu \mathrm{m}$, and the position of the object on the $J-H$ vs. $H-K$ diagram suggests a reddened background star with $A_{\mathrm{V}} \geq 15$ mag. The contribution of this source in the $3^{\prime}$ ISOPHOT beam can explain why the ISOPHOT $4.8 \mu \mathrm{m}$ point is higher than the $M$-band observation of 1983 . In the $12-25 \mu \mathrm{m}$ range the ISO fluxes are higher while the MSX fluxes are lower than the IRAS fluxes.
This result, however, can be explained by the presence of the arcminute core associated to V1515 Cyg, taking into account the different beam sizes of the three instruments (MSX: 18", IRAS: 1', ISOPHOT: $\left.3^{\prime}\right)$. At near-infrared wavelengths the data indicate a slight $(\sim 15 \%)$ flux decrease, which is also visible in the $K$-band lightcurve (Fig. 3 ). In the mid-infrared the comparison of the 1983 and 1996-98 SEDs is complicated by the beam confusion. The fact, however, that at $\sim 20 \mu \mathrm{m}$ $Q$-band measurements from 1974 (4.1 $\pm 0.7 \mathrm{Jy}$, Cohen 1974) and 1989 (2.9 $\pm 0.6 \mathrm{Jy}$, Kenyon et al. 1991) agree with the MSX flux value of 3.0 $\pm 0.7 \mathrm{Jy}$ suggests that the mid-infrared fluxes of V1515 Cyg remained constant. At far-infrared wavelengths the emission of V1515 Cyg was constant within the measurement uncertainties.

V1735Cyg. The $850 \mu \mathrm{m}$ submillimetre map of Sandell \& Weintraub (2001) reveals a cold compact source, V1735 Cyg SM 1, at 20" from the star dominating the emission at both 450 and $850 \mu \mathrm{m}$. From a careful analysis of the MSX images we concluded that in the whole near- and midinfrared regime the submillimetre source is invisible and the observed emission can be assigned to V1735 Cyg. Thus, the star is responsible for the observed flux decrease of $\approx 40 \%$ below $5 \mu \mathrm{m}$, in accordance with the change in the $K$-band lightcurve (Fig. 3). No flux variation is visible at mid-infrared wavelengths. The relative contribution of the submm source, however, is probably increasing at longer wavelengths, as indicated by the fact that the IRAS position is located between the positions of the FU Orionis object and that of SM 1. Both the IRAS and ISOPHOT beams include both sources. Our result of no flux variation at far-infrared wavelengths indicates that the emission of V1735 Cyg was constant.

V346 Nor. This star is situated in a complex region including the PMS star Reipurth 13, HH 56/57 and some cold extended emission within a radius of 60" (Sandell \& Weintraub 2001). 


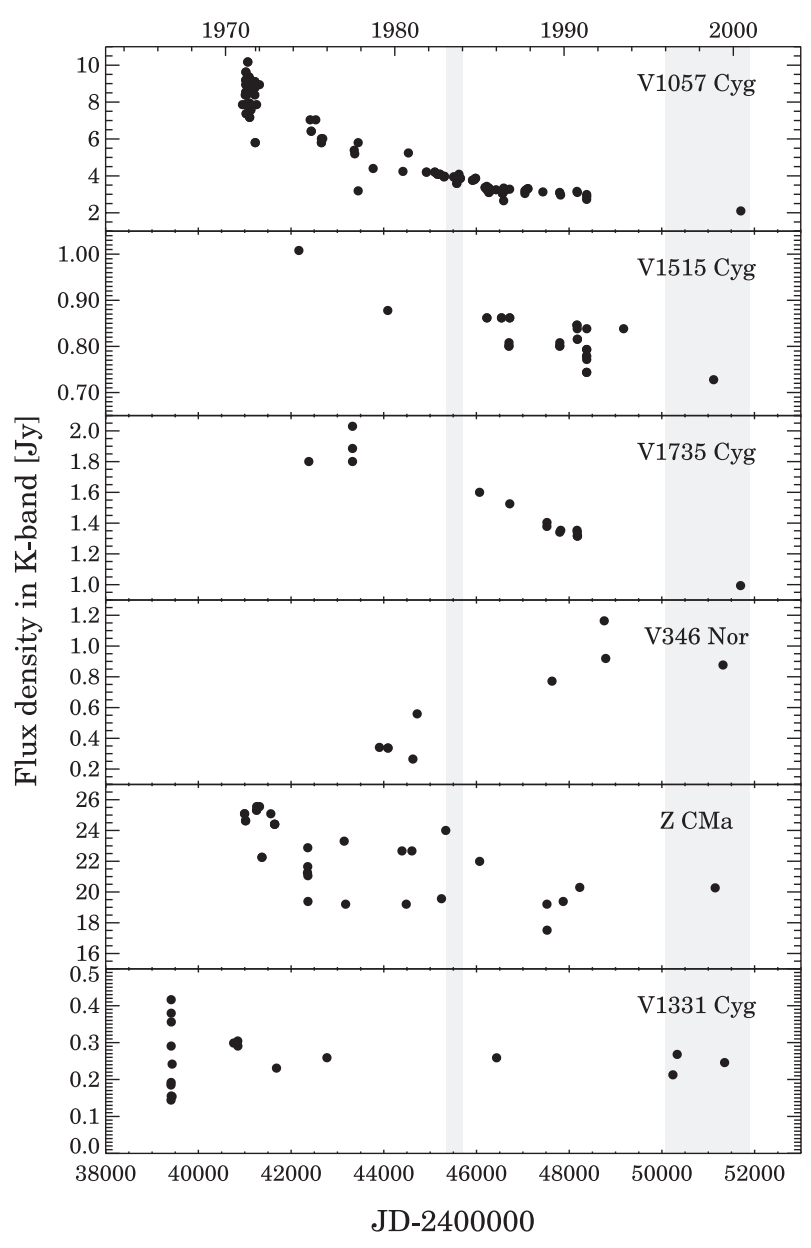

Fig. 3. $K$-band lightcurve of 6 FU Orionis objects for the period 1963-2003. Data are taken from the literature (for Parsamian 21 no observations are available). Grey stripes mark the two epochs (1983 and 1996-2000) which form the baseline for the long-term evolution.

Applying a maximum entropy based deconvolution technique, Prusti et al. (1993) were able to determine the relative contribution of Re 13 (separated by $\approx 50^{\prime \prime}$ from the FU Orionis star) in the IRAS bands, and found that its flux is a factor of 3-5 times lower than the emission of V346 Nor. At near-infrared wavelengths the FU Orionis star - uniquely in our sample - became brighter. This behaviour, reported already by Prusti et al. (1993), is also shown by the $K$-band lightcurve (Fig. 3). The brightening seems to be wavelength-dependent: its amplitude increases monotonically from $J$ to $K$. The same trend can still be observed at mid-infrared wavelengths up to $25 \mu \mathrm{m}$ where the IRAS and ISOPHOT fluxes are the same. On the other hand at $\lambda \geq 60 \mu \mathrm{m}$ the observed fluxes became lower between 1983 and 1996. Assuming that Re 13 (which is also included in the beam) is not variable we may conclude that V346 Nor faded at 60 and $100 \mu \mathrm{m}$. It is remarkable that the flux change is associated with a change in the far-infrared colour, too, which became warmer during the 15 years. The observed change could be an indication for a temperature rise in the outer parts of the circumstellar environment, but it might also be an artifact caused by the different sensitivities of the IRAS and ISOPHOT instruments for the extended emission.
ZCMa is a binary system with a separation of $0.1^{\prime \prime}$. The component which dominates at infrared wavelengths is probably a Herbig $\mathrm{Ae} / \mathrm{Be}$ star, while its optically brighter companion is an FU Orionis object. The system is embedded in an elongated disk-like submm core (Sandell \& Weintraub 2001), and was unresolved by both IRAS and ISOPHOT. According to the $K$-band lightcurve (Fig. 3) ZCMa did not change after 1974. The same conclusion can be drawn for the whole $2-100 \mu \mathrm{m}$ SED. This result, however, does not exclude a small flux variation of the FU Orionis component since the infrared SED of the system is dominated by the intermediate mass component.

Parsamian 21. This source is relatively isolated, no extended emission is associated with it at submillimetre wavelengths (Henning et al. 1998), thus beam differences of the different instruments are unimportant. The comparison of the two SEDs does not reveal any long term flux variation between 1983 and 1996-98 within the measurement uncertainties.

V1331 Cyg is believed to be in a state prior to an FU Orionistype outburst (Welin 1976; McMuldroch et al. 1993). The source is compact at submillimetre wavelengths (Henning et al. 1998), thus the comparison of the various observations is not influenced by beam effects. Our data show no evidence of temporal variation of the source's flux in the whole infrared regime. The $K$-band lightcurve (Fig. 3 ) is consistent with this conclusion.

\section{Discussion: The case of V1057 Cyg}

In this section we compare our new observational results on the temporal evolution of FU Orionis objects with predictions of models developed to describe their circumstellar structure. From our sample V1057 Cyg has the best documented multiwavelength flux evolution following its outburst in 1970. The ISO data points, supplemented by other infrared observations (Fig. 2), reveal a fast temporal evolution between $\sim 1983$ and 1996-2000 (the fastest rate in the sample). The wavelength dependence of the evolution is also well determined. Since detailed models of V1057 Cyg are available in the literature, we chose this object to compare the new observational results on the temporal evolution with model predictions.

As far as we know there are two models of the circumstellar environment of V1057 Cyg to fit the complete 1-100 $\mu \mathrm{m}$ infrared SED (Kenyon \& Hartmann 1991; Turner et al. 1997). Kenyon \& Hartmann (1991) collected and analysed all data available until 1991 and modelled the post-outburst evolution in the framework of an accretion disk model. They concluded that a simple accretion disk model cannot explain the large farIR fluxes, and investigated two possible geometries, a flared disk and an extended infalling envelope. Since the flared disk would require very high coverage factor, the authors favour a model where a flat disk is embedded in a spherically symmetric envelope with a wind-driven polar hole. The envelope, which is the remnant of the molecular cloud core, reprocesses the radiation from regions close to the central star. Turner et al. (1997) 
fitted the SED of V1057 Cyg by computing outbursting flared disk models in which the mass flux varies with radius. The model includes reprocessing of disk emission by other parts of the disk. An envelope with a central hole exposing the inner disk is included as a layer of uniform thickness on top of the disk.

At optical and near-IR wavelengths $(\lambda \leq 2.2 \mu \mathrm{m})$ both models claim that emission of the central source (the star plus the innermost part of the accretion disk) dominates the observed flux. After the outburst the accretion rate close to the star decreases, leading to a flux decrease in this wavelength range. Our data confirm that the flux decay was observable between 1983 and 2000 , too. The data reveal that in the $R, J, H$, and $K$ bands the flux dropped by the same constant factor of 2, while at $B$ and $V$ a larger decay was observed (lower panel of Fig. 2). This wavelength dependence may reflect the drop of effective temperature leading to a shift in the peak of the emission of the central source towards longer wavelengths.

Between 3 and $10 \mu \mathrm{m}$ the origin of the emission is the release of accretion energy in the disk (Kenyon \& Hartmann 1991), and also starlight reprocessed in the same part of the disk and in an envelope (Turner et al. 1997). A decreasing accretion rate at the center (which leads to a decrease in the bolometric luminosity) would cause the drop of emission of all three components. The timescale at which viscous accretion could change is related to the dynamical timescale at this distance (several years at $\sim 1$ AU, Pringle 1981). The timescale on which the reprocessed emission follows the fading of the central source depends on the optical depth of the reprocessing medium. For an optically thin medium (disk surface layer, envelope) an almost instantaneous reaction is expected (several hours, Chiang \& Goldreich 1997, Eq. (25)). For optically thick emission (disk interior) the thermal timescale related to the gas component dominates (several years at $\sim 1$ AU for a typical T Tauri star, Chiang \& Goldreich 1997, Eq. (28)). In the case of V1057 Cyg the $3-10 \mu \mathrm{m}$ emission is probably not completely optically thick, as indicated by the presence of weak spectral features (silicate emission at $9.7 \mu \mathrm{m}$, Wooden et al. 1995; Hanner et al. 1998). Since our temporal baseline (1983-1998) significantly exceeds the mentioned timescales and since the post-outburst evolution had already started in the seventies, the model prediction is that the $3-10 \mu \mathrm{m}$ emission is decreasing in synchronization with the rate of the optical/near-IR decay in a wavelength independent way. Our measurements are fully consistent with these predictions. (Fig. 2 lower panel)

The emission at $\lambda>10 \mu \mathrm{m}$ is reprocessed starlight in both the model of Kenyon \& Hartmann (1991) and of Turner et al. (1997). The infrared radiation emerges from an envelope where dust particles are in radiative equilibrium with the illumination from the central region (in the model of Turner et al. the envelope is optically thick to its own radiation at $\lambda \leq 30 \mu \mathrm{m}$ ). The temporal evolution implied by the models is similar to the predicted trend at shorter wavelengths: a wavelength independent fading at the same rate as the fading of the central illuminating source (although the time-lags are gradually longer at longer wavelengths, Chiang \& Goldreich 1997). Our results are not fully consistent with this picture. Though at $10 \mu \mathrm{m}$ the flux dropped between 1983 and 1996 by a factor of 2 similarly to the optical/near-IR rate, at far-IR wavelengths $(\lambda \geq 60 \mu \mathrm{m})$ the flux of V1057 Cyg remained constant. The comparison of the IRAS 60 and $100 \mu \mathrm{m}$ photometric points with our new ISOPHOT data at the same wavelengths clearly demonstrates that the far-infrared fluxes of V1057 Cyg showed no variation between 1983 and 1997. The transition between the two types of behaviour is around $25 \mu \mathrm{m}$.

Our results indicate that - unlike in the above mentioned models - at $\lambda \geq 10 \mu \mathrm{m}$ two important emission components have to be taken into consideration. Between 10 and $25 \mu \mathrm{m}$ we probably observe the envelope, while at longer wavelengths the emission cannot be explained this way. The material responsible for the $\lambda>25 \mu \mathrm{m}$ emission is apparently not an optically thin medium reprocessing the light of the central source, because its radiation does not react immediately on the source's fading. Moreover, the emitting material has the following properties: (1) it is relatively cold; (2) it has a flat spectrum below $100 \mu \mathrm{m}$, suggesting a $T \sim r^{-0.5}$ radial temperature profile.

The first possibility for the nature of the far-infrared emitting component could be a reprocessing medium which is optically thick (e.g. a flared disk), whose time-lag behind the fading of the central source significantly exceeds our temporal baseline. Kenyon \& Hartmann (1991), however, demonstrated that the outer part of a flared disk around V1057 Cyg would produce an unacceptably large covering factor and rejected this possibility. In addition, based on more detailed calculations, Bell et al. (1997) pointed out that such a flared structure would not be realised in nature. At a radius of $\sim 10 \mathrm{AU}$ the flared disk would develop into a "decreasing curvature" disk, corresponding to the condensation of water ice; thus the outer disk is shadowed from the illumination of the central source. Such a disk cannot produce either the $T \sim r^{-0.5}$ temperature profile or the high level of far-infrared emission. Based on these arguments we exclude reprocessing of the radiation of the central source in the outer part of the disk as the origin of the far-infrared emission.

Nevertheless, it should be mentioned that the critical radius where the disk turns to concave (i.e. the border line between the illuminated and shadowed parts of the disk) corresponds to a temperature of $\approx 300 \mathrm{~K}$ (Bell et al. 1997). Since the blackbody emission of this temperature contributes mainly to the $25 \mu \mathrm{m}$ range of the SED, one might speculate that the change in the temporal behaviour of the SED of V1057 Cyg, also at $25 \mu \mathrm{m}$, could be related to the turnover of the disk profile. However, in the previous paragraphs we concluded that neither the short wavelength emission ( $\lambda \leq 25 \mu \mathrm{m}$, attributed to an envelope) nor the long wavelength part of the SED are dominated by the radiation of an optically thick passive disk. We think that the agreement of the transition wavelength in V1057 Cyg at $25 \mu \mathrm{m}$ with the peak of the blackbody curve at the sublimation temperature of water ice in the disk is a coincidence. This conclusion is further supported by the case of V1735 Cyg, where the shortest wavelength of constant flux is at $10 \mu \mathrm{m}$, clearly different from the temperature of the turnover radius (Fig. 1).

As a second possibility for the origin of the far-infrared emission Bell et al. (1997) proposed that the heating of the outer part of an optically thick disk could be dominated by the ambient radiation field of the cloud core around the young star, rather than by the radiation of the central source. As a result, 
the radial temperature profile becomes constant at the ambient temperature value, producing a shallower - or even approximately flat - far-infrared SED. Since its origin is unrelated to the central source, the far-infrared emission is not expected to vary in time.

A third possibility would be that the far-IR emission is powered by accretion in the outer disk (active disk), where the accretion rate is constant and unrelated to the outburst of the central region (Bell et al. 1995). However, the spectral shape of such a standard accretion disk would follow the canonical $v F_{v} \sim \lambda^{-4 / 3}$ law, in contradiction with the observed flat spectrum (Fig. 2). A possible solution for this problem could be that the temperature profile of the accretion disk differs from the standard one. Lodato \& Bertin (2001) suggested that in a selfgravitating protostellar disk the non-Keplerian rotation curve may result in a non-standard temperature distribution, and thus in a flat spectrum in the far-IR. The high disk mass required by this model $\left(\approx 1 M_{\odot}\right)$ is not inconsistent with measurements of some FU Orionis objects (Henning et al. 1998; Sandell \& Weintraub 2001), but the measured disk mass of V1057 Cyg $\left(\approx 0.1 M_{\odot}\right.$, Sandell \& Weintraub 2001) seems to be too low to cause any observable effect.

The last possibility would be to assume that the origin of the far-IR emission is unrelated to V1057 Cyg. Such a source could be a - so far undetected - embedded IR companion. It is interesting to note that Bell et al. (1995) already speculated about such a companion which could have triggered the outburst of V1057 Cyg by a close passage. A deeply embedded infrared companion, like e.g. the very red object discovered in the vicinity of $\mathrm{LkH} \alpha 198$ at $10 \mu \mathrm{m}$ (Lagage et al. 1993), might be too faint to have been detected at near-infrared wavelengths so far. At mid- and far-infrared wavelengths V1057 Cyg has never been imaged with high-resolution instruments, thus the existence of a companion unresolved in the beam of the MSX $\left(18^{\prime \prime}\right)$ cannot be excluded.

In the previous paragraphs we argued that an optically thick disk, reprocessing the radiation of the central source, or accretion with a non-Keplerian rotation curve are less likely explanations, but a shallower temperature profile dictated by the ambient radiation field or the possibility of an unrelated infrared companion cannot be excluded. The observations (Fig. 1) suggest that the constancy of the far-infrared emission might be a general property of FU Orionis objects. Though a growing number of FU Orionis objects are found to be binaries (Reipurth \& Aspin 2004), it has not been proved yet that all FU Orionis objects have companions. Thus we think that the most likely origin of the far-infrared radiation in V1057 Cyg (and perhaps in other FU Orionis objects) is emission from the outer part of an optically thick disk whose temperature profile is controlled by the ambient radiation field.

Our investigation demonstrates that temporal variability of the infrared emission can provide important information on the physical properties of circumstellar material around FU Orionis objects. This kind of diagnostics of the disk structure - proposed first by Chiang \& Goldreich (1997) - can also be applied to any other type of young stellar object where the energy source of the disk, either accretion or reprocessing, is time-variable (e.g. the EX Lup-type eruptive young stars). New infrared data on some FU Orionis objects are expected from the Spitzer Space Telescope which could show whether the evolutionary trends outlined by the ISO data also continue in our days, and whether the variations of the SEDs at longer wavelengths are responding to the flux drop at shorter wavelengths.

Acknowledgements. The observations were reduced using the ISOPHOT Interactive Analysis package PIA, which is a joint development by the ESA Astrophysics Division and the ISOPHOT Consortium, lead by the Max-Planck-Institut für Astronomie (MPIA). This research has made use of the NASA/ IPAC Infrared Science Archive, which is operated by the Jet Propulsion Laboratory, California Institute of Technology, under contract with the National Aeronautics and Space Administration. This research has also made use of the SIMBAD database, operated at CDS, Strasbourg, France. We received comments and suggestions from L. Szabados and D. Apai who carefully read the manuscript. We are grateful to an anonymous referee who called our attention to models of the temperature and opacity structure in the outer parts of protostellar disks. The work was partly supported by the grant OTKA T 037508 of the Hungarian Scientific Research Fund. P.Á. acknowledges the support of the Bolyai Fellowship.

\section{References}

Aspin, C., \& Sandell, G. 2001, MNRAS, 328, 751

Aspin, C., \& Reipurth, B. 2003, AJ, 126, 2936

Bell, K. R., Lin, D. N. C., Hartmann, L. W., \& Kenyon, S. J. 1995, ApJ, 444, 376

Bell, K. R., Cassen, P. M., Klahr, H. H., \& Henning, Th. 1997, ApJ, 486, 372

Carr, J. S., Harvey, P. M., \& Lester, D. F. 1987, ApJ, 321, L71

Chavarria-K. C. 1981, A\&A, 101, 105

Chiang, E. I., \& Goldreich, P. 1997, ApJ, 490, 368

Cohen, M. 1974, PASP, 86, 813

Cutri, R. M., et al. 2003, Explanatory Supplement to the 2MASS All Sky Data Release (Pasadena: Caltech)

de Lara, E., Chavarria-K. C., \& Lopez-Molina, G. 1991, A\&A, 243 , 139

Egan, M. P., \& Price, S. D. 1996, AJ, 112, 2862

Eiroa, C., \& Hodapp, K.-W. 1990, A\&A, 236, 217

Eislöffel, J., Hessman, F. V., \& Mundt, R. 1990, A\&A, 232, 70

Elias, J. H. 1978, ApJ, 223, 859

Gabriel, C., et al. 1997, in Proc. of the ADASS VI conference, ed. G. Hunt, \& H. E. Payne, ASP Conf. Ser., 125, 108

Graham, J. A., \& Frogel, J. A. 1985, ApJ, 289, 331

Hanner, M. S., Brooke, T. Y., \& Tokunaga, A. T. 1998, ApJ, 502, 871

Hartmann, L., \& Kenyon, S. J. 1996, ARA\&A, 34, 207

Hartmann, L., Kenyon, S. J., Hewett, R., et al. 1989, ApJ, 338, 1001

Henning, Th., Burkert, A., Launhardt, R., Leinert, Ch., \& Stecklum, B. $1998, A \& A, 336,565$

Herbig, G. H. 1977, ApJ, 217, 693

Herbst, W., Racine, R., \& Warner, J. W. 1978, ApJ, 223, 471

Hodapp, K. W. 1995, IAU Circ., 6186, 2

Ibragimov, M. A., \& Shevchenko, V. S. 1988, Astrophysics (Tr. Astrofizika), 27, 337

Kenyon, S. J., \& Hartmann, L. 1991, ApJ, 383, 664

Kenyon, S. J., Hartmann, L., \& Kolotilov, E. A. 1991, PASP, 103, 1069

Kenyon, S. J., Hartmann, L., Gomez, M., Carr, J. S., \& Tokunaga, A. 1993, AJ, 105, 1505

Kessler, M. F., Steinz, J. A., Anderegg, M. E., et al. 1996, A\&A, 315, L27 
Kopatskaya, E. N. 1984, Astrofizika, 20, 163 (English translation: 1984, Astrophysics, 20, 138)

Lagage, P. O., Olofsson, G., Cabrit, S., et al. 1993, ApJ, 417, L79

Laureijs, R. J., Klaas, U., Richards, P. J., Schulz, B., \& Ábrahám, P. 2001, The ISO Handbook, vol. V, ESA SAI-99-069/Dc, Version 1.2

Lemke, D., Klaas, U., Abolins, J., et al. 1996, A\&A, 315, L64

Lis, D. C., Menten, K. M., \& Zylka, R. 1999, ApJ, 527, 856

Lodato, G., \& Bertin, G. 2001, A\&A, 375, 455

McMuldroch, S., Sargent, A. I., \& Blake, G. A. 1992, BAAS, 24, 1215

McMuldroch, S., Sargent, A. I., \& Blake, G. A. 1993, AJ, 106, 2477

Mitchell, G. F., Johnstone, D., Moriarty-Schieven, G., Fich, M., \& Tothill, N. F. H. 2001, ApJ, 556, 215

Moór, A., Ábrahám, P., Csizmadia, Sz., Kiss, Cs., \& Klaas, U. 2004, in preparation

Pringle, J. E. 1981, ARA\&A, 19, 137
Prusti, T., Bontekoe, Tj. R., Chiar, J. E., Kester, D. J. M., \& Whittet, D. C. B. 1993 , A\&A, 279, 163

Reipurth, B., \& Aspin, C. 1997, AJ, 114, 2700

Reipurth, B., \& Aspin, C. 2004, ApJ, 608, L65

Sandell, G., \& Aspin, C. 1998, A\&A, 333, 1016

Sandell, G., \& Weintraub, D. A. 2001, ApJS, 134, 115

Staude, H. J., \& Neckel, Th. 1991, A\&A, 244, L13

Staude, H. J., \& Neckel, Th. 1992, ApJ, 400, 556

Strom, K. M., \& Strom, S. E. 1993, ApJ, 412, L63

Tuffs, R., \& Gabriel, C. 2003, A\&A, 410, 1075

Turner, N. J. J., Bodenheimer, P., \& Bell, K. R. 1997, ApJ, 480, 754

Weaver, W. B., \& Jones, G. 1992, ApJS, 78, 239

Welin, G. 1976, A\&A, 49, 145

Wooden, D. H., Bell, K. R., Butner, H. M., \& Goguen, J. D. 1995, BAAS, 27, 1320

Yun, J. L., Moreira, M. C., Alves, J. F., \& Storm, J. 1997, A\&A, 320, 167 